\title{
SERPENT/SUBCHANFLOW COUPLED BURNUP CALCULATIONS FOR VVER FUEL ASSEMBLIES
}

\author{
Diego Ferraro ${ }^{1}$, Manuel García ${ }^{1}$, Uwe Imke ${ }^{1}$, Ville Valtavirta ${ }^{2}$, Riku Tuominen ${ }^{2}$, Jaakko \\ Leppänen ${ }^{2}$ and Víctor Sanchez-Espinoza ${ }^{1}$ \\ ${ }^{1}$ Karlsruhe Institute of Technology, Institute of Neutron Physics and Reactor Technology, \\ Hermann-von-Helmholtz-Platz 1, 76344 Eggenstein-Leopoldshafen, Germany \\ ${ }^{2}$ VTT Technical Research Centre of Finland Ltd., P.O. Box 1000, FI-02044 VTT, Finland \\ diego.ferraro@kit.edu
}

\begin{abstract}
The continuous improvement in nuclear industry safety standards and reactor designers' and operators' commercial goals represent a push for the development of highly accurate methodologies in reactor physics. This fact, combined with the availability of vast computational resources, allowed the development of a wide range of coupled state-of-the-art neutronic-thermal-hydraulic calculation tools worldwide during last decade. Under this framework, the McSAFE European Union project is a coordinated effort aimed to develop multiphysics tools based on Monte Carlo neutron transport and subchannel thermal-hydraulics codes, suitable for high-fidelity calculations for PWR and VVER reactors. This work presents the results for a pin-by-pin coupled burnup calculation using the Serpent 2 code (developed by VTT, Finland) and the subchannel thermal-hydraulics code SUBCHANFLOW (SCF, developed by KIT, Germany) for two different VVER-type fuel assembly types. For such purpose, a recently refurbished master-slave coupling scheme is considered, which provides several new features such as burnup and transient calculations capabilities for square and hexagonal geometries. Main aspects of this coupling are presented for this burnup case, showing some of the capabilities now available. On top of that, the obtained global results are compared with available published data from a similar high-fidelity approach for the same FA design, showing a good agreement. Finally, a brief analysis of the main resources requirement and main bottlenecks identification are also included. The results presented here provide valuable insights and pave the way to tackle the final goals of the McSAFE project, which includes full-core pin-by-pin depletion calculation within a fully coupled MC-TH approach.
\end{abstract}

KEYWORDS: VVER, Serpent, SCF, codes coupling, burnup, high-fidelity

\section{INTRODUCTION}

During the last decade, a global trend for the development of highly accurate methodologies in reactor physics has been observed in several projects worldwide [1,2], where the availability of vast computational resources allows the development of a wide range of coupled state-of-the-art neutronic and thermalhydraulic $(\mathrm{TH})$ calculation tools. These analyses, usually identified as high-fidelity, tend to diminish the level of approximations in the involved coupled physics through the full-scope coupling of codes with high detail modelling capabilities. Under this framework, the McSAFE European Union project [2] is a coordinated effort aimed to develop multiphysics tools based on Monte Carlo (MC) neutron transport and 
subchannel TH codes, suitable for high-fidelity calculations for PWR and VVER reactors for steady-state, burnup and transient calculations. Regarding burnup calculations, one of the project stated goals is to develop full-core depletion with TH coupling at pin level, which represents a challenging target.

For this purpose, a series of tools have been developed, which rely on the coupling through diverse methods $[6,7]$. The proper verification and validation of these schemes in a wide range of geometries is compulsory, together with the performance analysis and the identification of potential bottlenecks. For practical reasons, this verification analysis is to be done step-by-step, where each stage provides valuable insights for the whole work path.

In this framework, the coupled burnup capability is tested for a 3D radial reflected FA. For such purpose a master-slave coupling between the MC Serpent 2 and the subchannel TH SUBCHANFLOW (namely SCF) codes is considered, where a full scope pin-by-pin coupling with burnup is developed for two VVER-type TVSA fuel assembly (FA) designs [8]. These proposed cases were already analyzed by similar high-fidelity schemes [9], thus providing reference global results for the whole burnup scope.

As a first step, the obtained Serpent-SCF global results are compared with reported ones from a similar high-fidelity approach [9]. These reference results were calculated using an MC neutronic code (namely MCU [8]), coupled with diverse TH modules, where the feedback of the coolant density and temperatures are obtained with a FA-level model (with 30 axial zones) while the fuel temperatures are managed through the fuel performance code TOPRA [8]. As a second step, in view of the stated high-fidelity goals, a series of detailed analysis of the results obtained with the Serpent-SCF approach are presented as an exemplification of the potential capabilities, including also a resource requirements analysis for this scheme.

\section{BENCHMARK CASE: VVER FA BURNUP WITH TH FEEDBACK}

Two VVER TVSA-type design FA are considered, both with enriched $\mathrm{UO}_{2}$ pins with and without burnable poisons (in the form of diluted Er oxide in pins), which will be identified as $U$-Er and $U$ respectively. These FA are to be calculated considering the TH feedback for a rated power for a typical VVER core and a proper burnup scope. The main data for both FA is presented in Table I, together with TH data in Table II.

Table I: Main characteristics of the FA proposed. Details can be gathered from [9].

\begin{tabular}{|c|c|c|}
\hline Parameter & $U$ & U-Er \\
\hline Fuel height [cm] & \multicolumn{2}{|c|}{368} \\
\hline Fuel rods number & \multicolumn{2}{|c|}{313} \\
\hline U Enrichment [\%wgt] & \multicolumn{2}{|c|}{4.4} \\
\hline Fuel assembly pitch [cm] & \multicolumn{2}{|c|}{23.6} \\
\hline Fuel rod pitch [cm] & \multicolumn{2}{|c|}{1.275} \\
\hline Fuel pellet diameter [cm] & \multicolumn{2}{|c|}{0.76} \\
\hline Fuel rod diameter[cm] & \multicolumn{2}{|c|}{0.91} \\
\hline Guide tube diameter[cm] & \multicolumn{2}{|c|}{1.26} \\
\hline BP in pins $\left(\mathrm{Er}_{2} \mathrm{O}_{3}\right)[\% \mathrm{wt}]$ & 0.0 & 1.0 \\
\hline
\end{tabular}

Table II: Main TH data. Details can be gathered from [9].

\begin{tabular}{|c|c|c|}
\hline Parameter & $U$ & $U-E r$ \\
\hline FA power $[\mathrm{kW}]$ & \multicolumn{2}{|c|}{18405} \\
\hline Coolant flow $\left[\mathrm{m}^{3} / \mathrm{h}\right]$ & \multicolumn{2}{|c|}{534} \\
\hline
\end{tabular}




\begin{tabular}{|c|c|}
\hline Pressure $[\mathrm{MPa}]$ & 15.6 \\
\hline Tinlet $[\mathrm{K}]$ & 560 \\
\hline
\end{tabular}

\section{COUPLING SCHEME}

As mentioned, a coupling between Serpent MC [4] and SCF subchannel TH codes [3] is considered, where the scheme selected for this work relies in a master-slave approach, recently rebuild from scratch to tackle burnup and transient calculations for a wide range of reactor geometries [7].

\subsection{Serpent 2}

Serpent 2 is a multi-purpose three-dimensional continuous energy Monte Carlo transport code, developed since 2004 at VTT Technical Research Centre of Finland Ltd. It represents a state-of-the-art code, aimed to perform static, burnup and dynamic 3-D calculations using standard ACE format Nuclear Data Libraries (NDL). It counts with several geometry definition alternatives, including the traditional 3D constructive solid geometry model and unstructured mesh geometries, which allows to deal with almost all reactor geometries usually encountered. Burnup capabilities are inherent, allowing an easy-handling approach. Besides, its particle tracking relies on the combination of conventional based surface tracking and the deltatracking method, improving global performance. The code also counts with inherent multi-physics features, namely due to the combined capability to handle variable material densities fields (through methods based on rejection sampling techniques) and also manage temperature fields through a rejection sampling approach combined with Target Motion Sampling. These capabilities can be easily used with the definition of a mesh that superimpose such TH fields in the geometry (namely multiphysics Interface Files - IFC) [5].

\subsection{SCF}

SUBCHANFLOW (SCF) is a subchannel-level thermal-hydraulic code for steady-state and transient calculations developed at KIT (Germany) [3]. The flow solver consists of four conservation equations, namely for mass, energy, and axial and lateral momentum. The main geometry is defined as a set of channels and rods with given hydraulic parameters and connectivities in a flexible manner, i.e. without assuming a particular geometry type such as square or hexagonal. In addition, a geometry preprocessor allows user to develop suitable inputs for PWR and VVER geometries through a high-level definition of lattices and geometry aspects.

\subsection{Coupling Serpent and SCF with burnup}

The coupling between both codes maintain all inherent burnup capabilities Serpent [4]. Besides, from SCF point of view, each burnup step represents a steady-state calculation, where the N-TH iteration is done according to Serpent selected scheme. For this work, a Predictor-Corrector scheme was selected with a TH feedback iteration on each step. A relaxation on TH fields between steps is done in terms of Equation (1):

$$
\tilde{T}_{i, j, k}^{t}=T_{i, j, k}^{t-1} w+T_{i, j, k}^{t}(1-w)
$$

where $\mathrm{T}_{\mathrm{i}, \mathrm{j}, \mathrm{k}}^{\mathrm{t}}$ represents the $\mathrm{TH}$ field in zone $\mathrm{i}, \mathrm{j}, \mathrm{k}$ for iteration $\mathrm{t}$ and $\mathrm{w}$ the relaxation factor. In addition a volume average of the fuel temperature in the pin is considered for the feedback, while a $\mathrm{L}_{2}$ norm is used for the convergence of the $\mathrm{TH}$ parameters for each by $\mathrm{i}, \mathrm{j}, \mathrm{k}$ zone. The convergence of the $\mathrm{TH}$ fields is set to $10 \mathrm{~K}$ and $2.5 \mathrm{~K}$ for fuel and coolant temperatures, while a $0.01[\mathrm{~g} / \mathrm{cm} 3]$ value is set for the coolant density variation. 


\section{MODELS DEVELOPED}

\subsection{Serpent and SCF models}

The models for Serpent were developed for both FA types in Table I. These considered a reflected boundary except for the bottom and top zones, where homogenized reflector compositions were included, followed by black boundary conditions. The JEFF3.11 NDL and equilibrium xenon were considered for both cases. Besides, a regular hexagonal fission power detector [11] with axial dependence at pin level was included for each case.

As an example, Figure 1 shows the model for the $U$ fuel case. It should be noted that standard TVSA stiffeners and grid spacers were considered [10] and a division of fuel zones by pin with 27 axial zones is considered. In addition, for the $U-E r$ case, two radial zones by pin were modelled. These totalized 8451 and 16902 burnable zones for $U$ and $U-E r$ cases respectively, which demanded $\sim 30$ GB RAM in the lowest optimization mode of Serpent $[11,12]$ for the most demanded case.

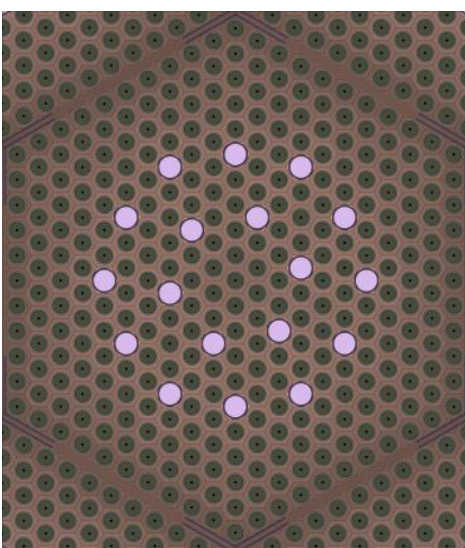

a) $x-y$ cut in the FA centre.

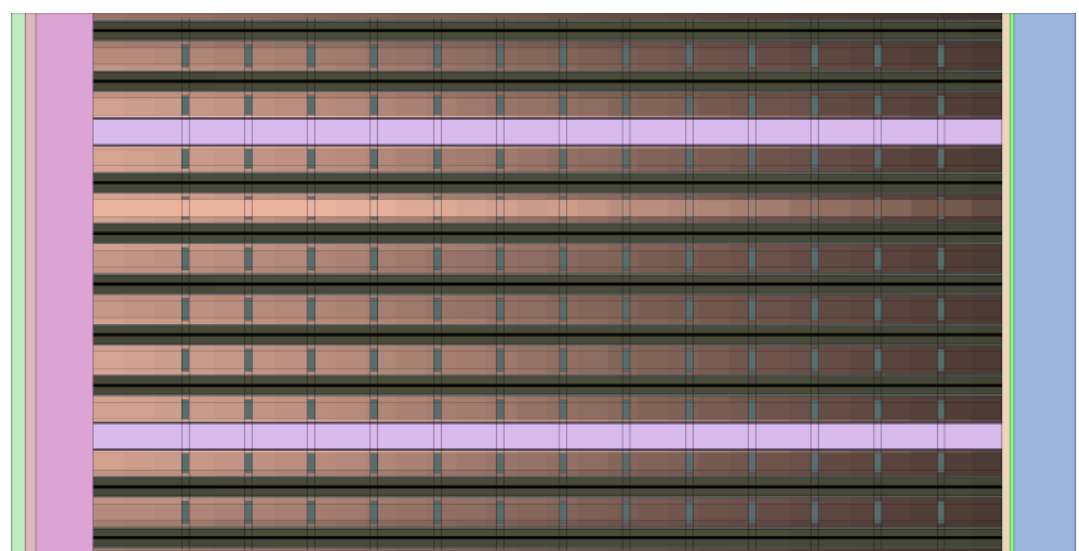

b) $z-y$ cut in the FA centre (not at scale)

Figure 1 Serpent model example

A fuel-centered channel level model [6] was developed in SCF, as show in Figure 2, where 30 axial zones were considered. This model was coupled with Serpent using ad-hoc mapping files that map one-to-one TH fields to proper nested hexagonal IFC [11]. The convergence of SCF fields was set to be at least two orders of magnitude below the N-TH coupling convergence of the fields.

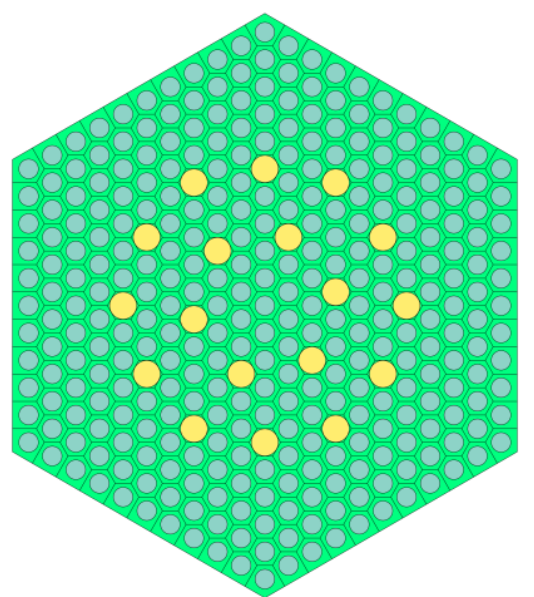

Figure 2 SCF model 
Finally, regarding Serpent statistical convergence, 2000 active cycles of 50000 particles each were considered (totalizing 1e8 active histories per step), obtaining a statistical convergence of $\sim 10 \mathrm{pcm}$ per step. In accordance with these values, the convergence of reactivity between $\mathrm{N}-\mathrm{TH}$ iterations was set to $\sim 35 \mathrm{pcm}$.

\section{RESULTS}

\subsection{Comparison of global results with reported data for $U$ and $U$-Er FA}

The results for the effective multiplication factors for both cases are presented in Figure 3. A comparison with the reported ones [9] (obtained with MCU+TH modules, referred as MCU-TOPRA) is included, presented as relative differences in reactivity (in pcm).

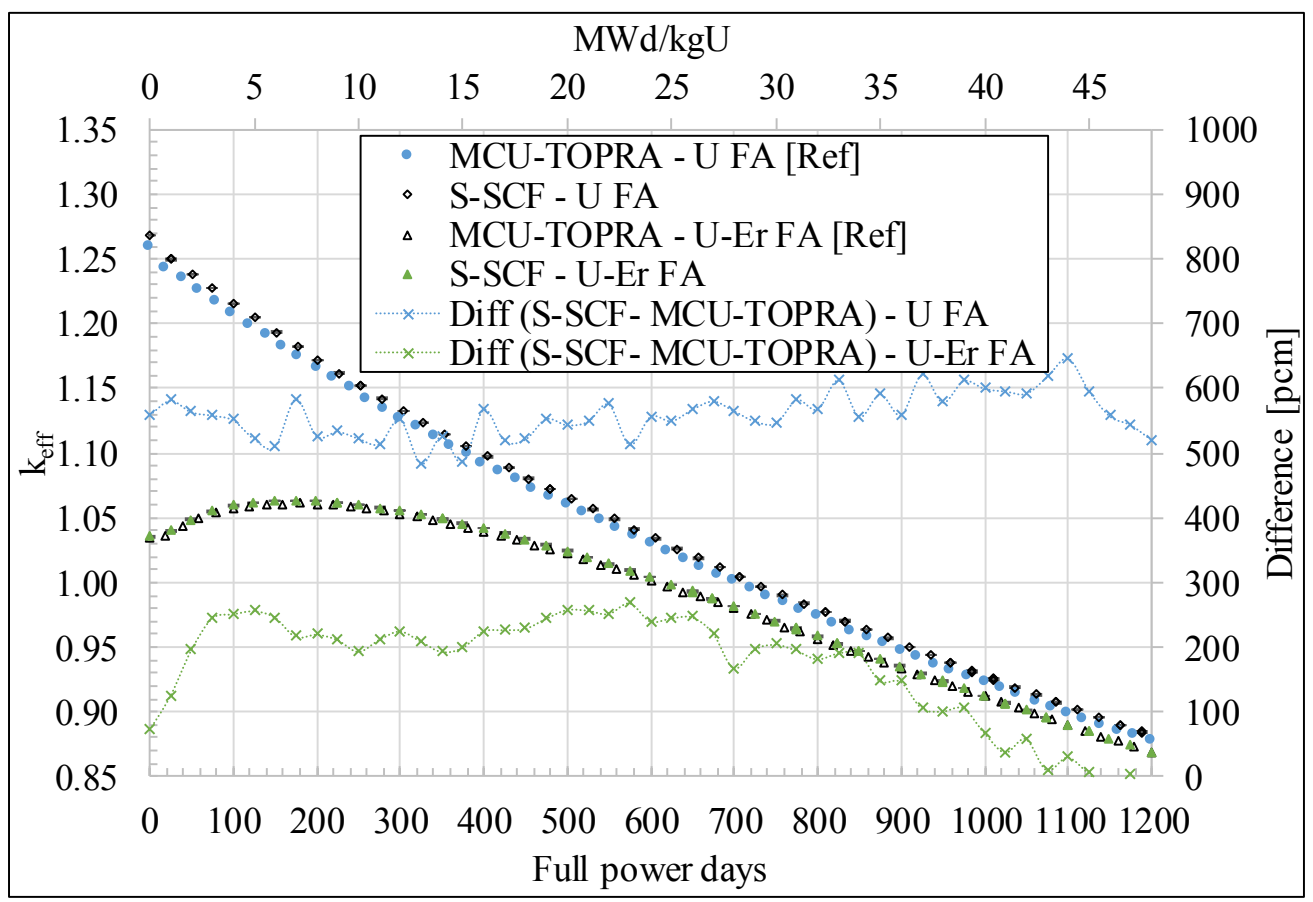

Figure 3 Comparison of effective multiplication factors for $\boldsymbol{U}$ and $\boldsymbol{U}$-Er fuels with reported values.

It can be seen from Figure 3 that a good global agreement is obtained, where average differences below $\sim 600 \mathrm{pcm}$ are observed for both cases. In addition the global behavior of the $\mathrm{k}_{\text {eff }}$ against burnup show a consistent evolution for both cases, where the burnup of the burnable poison is correctly reproduced.

\subsection{Towards high fidelity: highly detailed results with burnup dependence}

On top of the comparison of global results, the main goal of the proposed approach is to count with highly detailed results. To show these capabilities, power and TH field evolution examples are presented here. On the one hand, the Figure 4 presents the power density and temperatures by pin for a cut near the centre of the $U-E r$ FA for two burnup steps. On the other hand, Figure 5 shows the evolution with burnup of the axial power density profile for the central pin for both FA. It can be observed from both figures that all parameters behave as expected and highly detailed results are obtainable. In view of these results, further applications could be easily foreseen, such as detailed analysis for specific cases involving both TH coupling and burnup. 

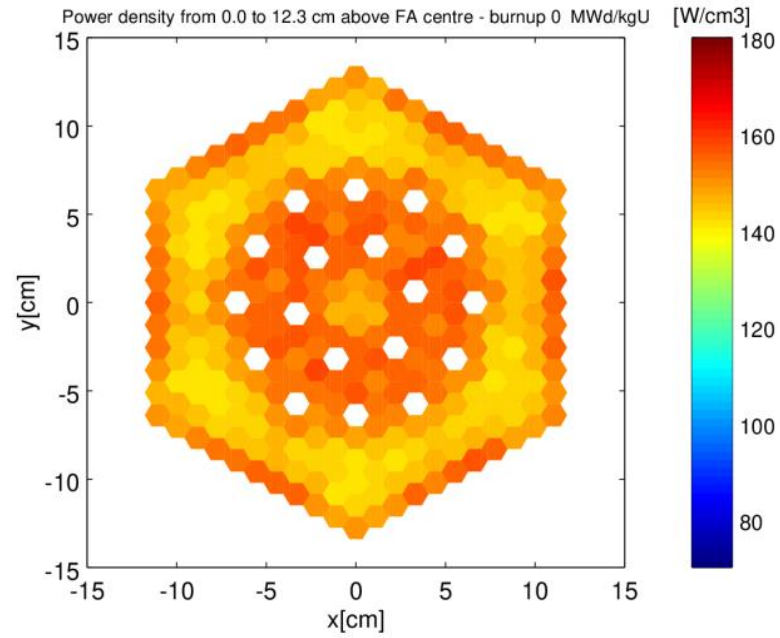

a) Power by pin in a $12 \mathrm{~cm}$ axial slice at $0 \mathrm{Mdw} / \mathrm{kgU}$
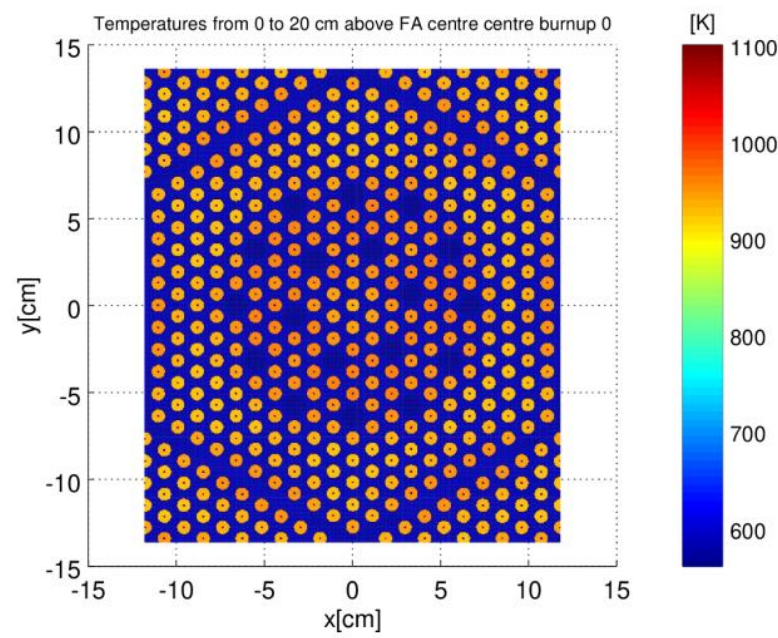

c) Temperatures in a $20 \mathrm{~cm}$ axial slice at $0 \mathrm{Mdw} / \mathrm{kgU}$

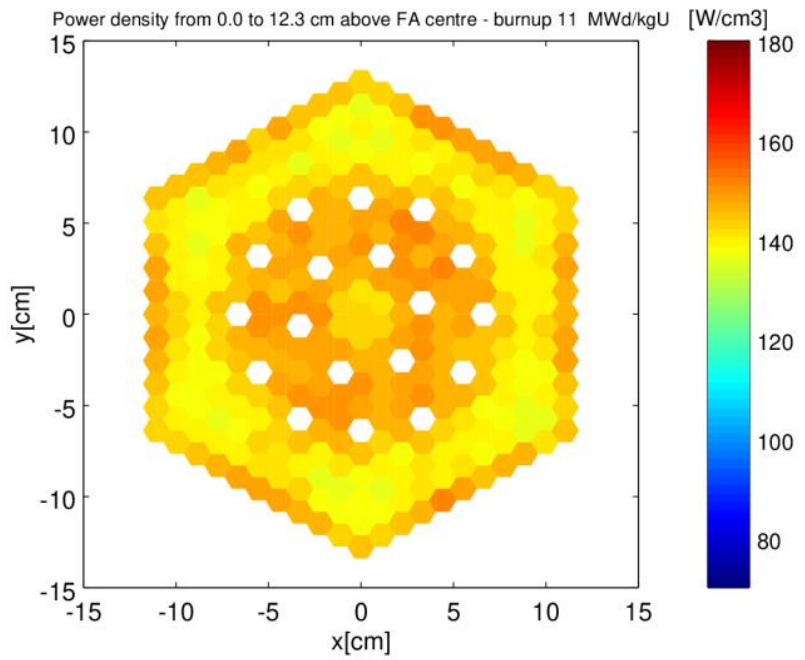

b) Power by pin in a $12 \mathrm{~cm}$ axial slice at $11 \mathrm{Mdw} / \mathrm{kgU}$

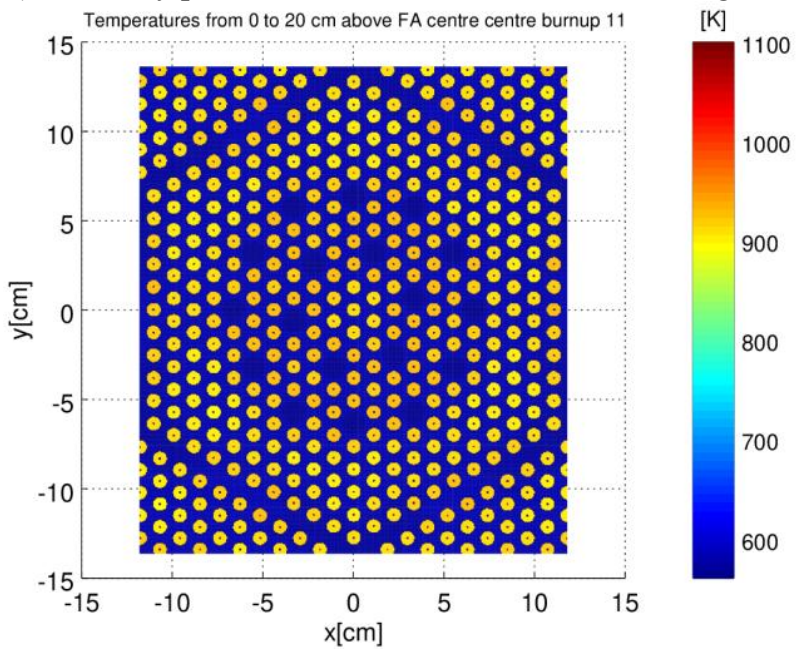

d) Temperatures in a $20 \mathrm{~cm}$ axial slice at $11 \mathrm{Mdw} / \mathrm{kgU}$

Figure 4 Evolution of power density and temperatures for an axial slice of $U$-Er FA.

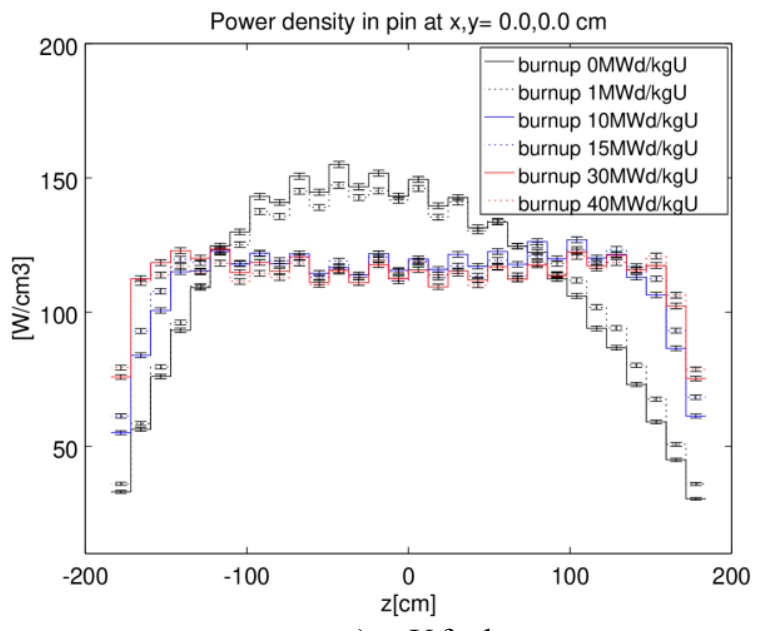

a) $U$ fuel

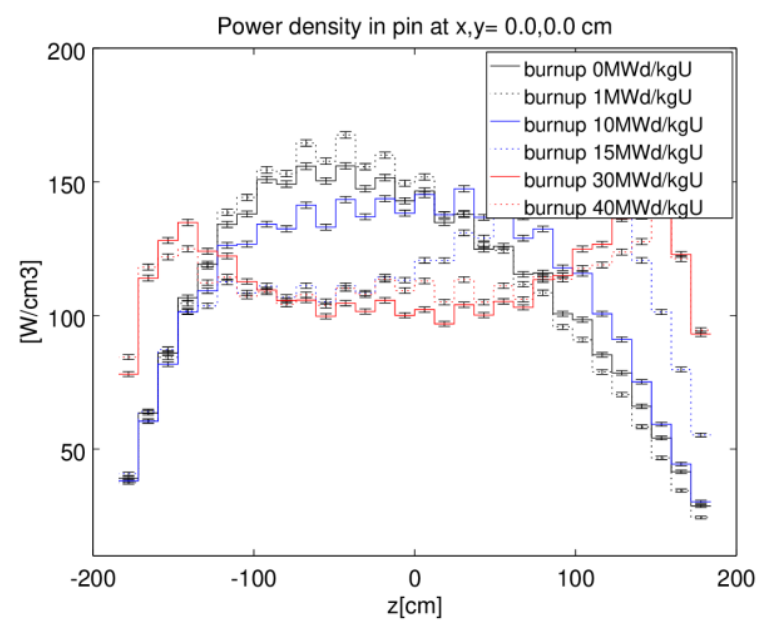

b) $U-E r$ fuel

Figure 5 Evolution of axial power profiles with burnup for selected pins in both FA. 


\subsection{Potential drawbacks and limitations: main resources requirement analysis}

As previously mentioned, the main drawback of these high-fidelity approaches is related to the amount of computational resources involved. A brief summary of average requirements for the $3 \mathrm{D}$ burnup problems solved is presented in Table III, using the $U$-Er FA case as reference. It should be noted here that these average values could also be used to develop estimations for further models where the overall size is bigger, such as a full-core or minicore-level problems.

Table III: Average running aspects of the proposed coupled scheme.

\begin{tabular}{|c|c|c|c|c|c|c|}
\hline \multicolumn{2}{|c|}{$\begin{array}{c}\text { Running time per } \\
\text { iteration step[mins] }\end{array}$} & \multicolumn{2}{|c|}{$\begin{array}{c}\text { Serpent average statistical } \\
\text { convergence }(1 \sigma)\end{array}$} & $\begin{array}{c}\text { Average number of } \\
\text { N-TH iterations per } \\
\text { burnup step }\end{array}$ & $\begin{array}{c}\text { RAM } \\
\text { memory [GB] }\end{array}$ \\
\cline { 1 - 5 } Serpent & SCF & $\begin{array}{c}\text { Reactivity } \\
\text { [pcm] }\end{array}$ & $\begin{array}{c}\text { Power by pin (30 axial } \\
\text { zones) max/ave [\%] }\end{array}$ & $\begin{array}{c}\text { Predictor } \\
\text { steps }\end{array}$ & $\begin{array}{c}\text { Corrector } \\
\text { steps }\end{array}$ & $\sim 30$ \\
\cline { 1 - 5 } $1.25 \mathrm{E}+04$ & $1 \mathrm{E}-01$ & $12 \mathrm{pcm}$ & $1.7 / 0.8$ & 2.4 & 2.2 & \\
\hline
\end{tabular}

${ }^{1} @$ Intel(R) Xeon(R) Gold 5118 CPU @ 2.30GHz

The analysis of Table III shows that the amount of computational resources is very high, directly related to the MC approach of the neutronics. In spite of this, it should be noted that the low number of N-TH iterations for both predictor and corrector steps is a strong indicator that is showing that the N-TH iteration could be done for larger steps than those used for burnup.

\section{CONCLUSIONS AND FURTHER WORK}

Following the global trend for the development of highly accurate methodologies in reactor physics, the McSAFE project developed several tools to tackle these high-fidelity goals. A proper verification and validation of these schemes in a wide range of geometries is compulsory, providing also valuable insights for the global performance of the approach and allowing the identification of potential bottlenecks.

The results presented within this work showed the combined capabilities of the proposed high-fidelity approach based on a recently refurbished S-SCF master-slave coupling. The consistency was assessed by modelling the burnup of a pin-by-pin N-TH coupled 3D-reflected VVER FA. The global comparison with reported values (calculated with a similar high-fidelity approach) showed a good agreement for the whole burnup scope. Besides, highly detailed results were also analyzed, showing a consistent behavior of the interchanged fields. Finally, a brief analysis of the computational resources requirement was also provided, showing one of the main drawback of these high-fidelity approaches, as far as the extrapolation to full-core calculations foresees the requirement of vast resources.

The results presented here provide valuable insights and pave the way to tackle the final goals of the McSAFE project. As a result, further works will deal with full-core VVER pin-by-pin depletion calculation within a fully coupled MC-TH approach.

\section{ACKNOWLEDGMENTS}

This work was done within the McSAFE project, which is receiving funding from the Euratom research and training programme 2014-2018 under grant agreement No 755097.

\section{REFERENCES}


1. "CASL - a DOE Energy Innovation Hub for Modeling and Simulation of Nuclear Reactors" The Consortium for Advanced Simulation of Light Water Reactors, https://www.casl.gov/.

2. L. Mercatali, et. al. "The EC McSAFE Project: High Performance Monte Carlo Methods for Safety Demonstration - Status and Perspectives" International Multi-Physics Validation Workshop, North Carolina State University, Raleigh, USA, June 14-15 (2018).

3. U. Imke, et. al. "Validation of the Subchannel Code SUBCHANFLOW Using the NUPEC PWR Tests (PSBT)". Science and Technology of Nuclear Installations, 2012.

4. Leppänen, J., et al. (2015) "The Serpent Monte Carlo code: Status, development and applications in 2013." Ann. Nucl. Energy, 82, pp. 142-150, (2015).

5. Valtavirta, V. "Internal Neutronics-temperature coupling in Serpent 2 -- Reactivity differences resulting from choice of material property correlations." In proc. M\&C 2013, Sun Valley, ID, May 5-9, 2013.

6. M. García, et al., Advanced Modelling Capabilities for Pin-level Subchannel Analysis of PWR and VVER Reactors, in: 18th International Topical Meeting on Nuclear Reactor Thermal Hydraulics (NURETH-18), 2019.

7. D. Ferraro et al. "Serpent/SUBCHANFLOW pin-by-pin coupled transient calculations for a PWR minicore" Ann. Nucl. Energy, 137, (2020).

8. Aleshin, S et al. "Calculations of 3D full-scale VVER fuel assembly and core models using MCU and BIPR-7A codes" Kerntechnik. 80. Pp. 326-338. (2015).

9. Bikeev, Artem \& Kalugin, Mikhail \& Oleinik, Dmitry \& Shcherenko, Anna \& A Shkarovsky, D \& Y Tomilov, M. "MCU Code Precision Calculation of a Full-Scale VVER-1000 Model Considering Feedbacks". Proceedings of International Conference on Mathematics \& Computational Methods Applied to Nuclear Science \& Engineering, At Jeju, Korea (2017).

10. Loetsch, T., Khalimonchuk, V., \& Kuchin, A. "Corrections and additions to the proposal of a benchmark for core burnup calculations for a WWER-1000 reactor" Proceedings of the twentieth symposium of atomic energy research, (p. 790). Hungary: Kiadja and KFKI Atomenergia Kutatointezet (2010).

11. Serpent developer team, Serpent Wiki on-line user Manual for Serpent 2, http://serpent.vtt.fi/mediawiki/index.php/Main_Page

12. Leppänen, J., \& Isotalo, A. "Burnup calculation methodology in the Serpent 2 Monte Carlo code". In Proceedings: International Conference on the Physics of Reactors 2012: Advances in Reactor Physics, PHYSOR 2012. ANS Vol. 2, pp. 924-935 (2012). 